\title{
Comparing prevalence and characteristic of anemia in children with simple versus complex febrile seizures
}

\author{
ALI AKBAR MOMEN ${ }^{1, A-C, E}$, ALI AMIN ASNAFI', B, ALI NIKKHAH ${ }^{3, B}$, MOHAMMAD REZA \\ KHALILIAN ${ }^{4, C}, \mathrm{D}, \mathrm{F}, \mathrm{MOHAMMAD} \mathrm{MAHMUDI}{ }^{5, C, F}$, HANNANEH DAVOODZADEH ${ }^{6, E, F}$
}

\author{
${ }^{1}$ Department of Pediatrics, Golestan Hospital, Ahvaz Jundishapour University of Medical Sciences, Ahvaz, Iran \\ ${ }^{2}$ Research Center of Thalassemia \& Hemoglobinopathy, Ahvaz Jundishapur University of Medical Sciences, Ahvaz, \\ Iran \\ ${ }^{3}$ Department of Pediatrics, Golestan Hospital, Ahvaz Jundishapour University of Medical Sciences, Ahvaz, Iran \\ ${ }^{4}$ Department of Pediatric Cardiology, School of Medicine, Ahvaz Jundishapur University of Medical Sciences, \\ Ahvaz, Iran \\ ${ }^{5}$ Student Research Committee, Ahvaz Jundishapur University of Medical Sciences, Ahvaz, Iran \\ ${ }^{6}$ Department of Toxicology, Islamic Azad University, Shahreza Branch, Shahreza, Isfahan, Iran
}

A - Study Design, B - Data Collection, C - Statistical Analysis, D - Data Interpretation, E - Manuscript Preparation, F - Literature Search, G - Funds Collection

\begin{abstract}
Summary Background. Febrile Convulsion (FC) disease is a seizure disorder associated with a febrile disease. In this study the relationship between incidence of anemia, particularly iron deficiency anemia, and FC has been evaluated.

Objectives. The aim of this study is to compare prevalence and characteristic of anemia in Simple and Complex Febrile Seizures in children referred to the Ahvaz Golestan Hospital, Iran.

Material and methods. This study was prepared by reviewing the medical records of patients with FC diseases, referred to the pediatrics department of Golestan Hospital between the years 2013 \& 2014. The information was entered into a check list, which included demographic, clinical and lab result data of patients. All data sets were statistically analyzed with the spss v22 program.

Results. Of the 207 patients, 151 patients (72.9\%) had simple seizures, and 56 patients (27.1\%) had complex febrile seizures. Average $\mathrm{MCH}$ in patients with simple and complexes FC was respectively $24.89 \mathrm{Pg}$., and $23.98 \mathrm{Pg}$.; and their difference were statistically significant $(p=0.03)$. The mean platelet count, in patients with simple FC was $329.01 / \mu \mathrm{L}$, and $273.38 / \mu \mathrm{L}$ in complex $\mathrm{FC}$ patients. The statistical difference was significant $(p=0.001)$. Regarding hemoglobin, hematocrit, $\mathrm{MCV}, \mathrm{RDW}$ and RBC there was no significant difference between simple and complex FC.

Conclusions. Given the significant difference of the average cell hemoglobin and platelet count in patients with simple and complex FC, a distinction must be made based on seizure type, in treatment of these patients.

Key words: seizures, febrile, anemia, hemoglobin, platelet.
\end{abstract}

Momen AA, Amin Asnafi A, Nikkhah A, Khalilian MR, Mahmudi M, Davoodzadeh H. Comparing prevalence and characteristic of anemia in children with simple versus complex febrile seizures. Fam Med Prim Care Rev 2018; 20(1): 25-28, doi: https://doi.org/10.5114/ fmpcr.2018.73700.

\section{Background}

Diseases of childhood are important areas in medical research. This age group need more attention compared to other age periods, as the diagnosis, treatment and management of these patients can have more effect on public health in long term. Among childhood diseases, seizure disorder is one of important ones, that needs more research and attention [1, 2]. Febrile seizure is one of the most common seizures in childhood [3]. According to the International League Against Epilepsy, febrile seizure or febrile convulsion (FC) is defined as a seizure associated with a febrile disease in the absence of a central nervous system infection or acute impairment of electrolyte balance in children aged older than one month with no history of non-febrile seizures $[4,5]$. FC was distinguished in the mid-nineteenth century from other types of convulsions [6]. FC mostly occurs between the ages of 6 months to 5 years, with a peak of incidence at the age of approx. 18 months $[3,5]$. The prevalence of $\mathrm{FC}$ has been reported to be $2-5 \%$, and the ratio of affliction in boys and girls is 1.4 to 1 [7]. FC occurs during fever caused by a viral or bacterial infection, such as respiratory tract infections or otitis media, when the body temperature is rapidly increasing. This is due to the inadequacy of the immature brain system of children in dealing with the stress of high fever [8]. Nelson and Ellenberg, using the data from the Joint National Perinatal Project, divided FC into two types: simple and complex [9]. Simple FC refers to generalized seizures lasting less than 15 minutes and which do not recur within 24 hours, while complex FC is defined as focal seizures which may last more than 15 minutes or recur within 24 hours [10]. Children with simple FC do not show evidence of increased mortality, hemiplegia or mental disability. During the follow-up of these patients, the risk of epilepsy after simple FC was found to be slightly higher than in the general population, and the main risk in these patients was a recurrence in one-third of them. Therefore, it can be concluded that simple FC is a benign condition with an excellent prognosis $[9,11]$. About $65-90 \%$ of FC seizures are of the simple type [7]. The primary risk factors for the first FC episode include genetic factors, presence in care centers, developmental delay, history of FC among the first- or second-degree relatives, staying in nursing centers for more than 30 days, maternal smoking during pregnancy and possibly iron and zinc deficiencies [10, 12 , 
13]. Children who have 2 of these 4 risk factors are $28 \%$ more likely to experience FC at least once [7]. Many studies have been conducted on the risk factors and etiology of FC, including the relationship between anemia and FC. Several studies have reported an association between iron-deficiency anemia and FC [14-17]. According to the World Health Organization (WHO), the prevalence of global anemia in the period 1993-2005 was $25 \%$, of which $44.7 \%$ was related to preschoolers [18]. In Iran, it is estimated that $20 \%$ of children suffer from anemia [12]. Iron-deficiency anemia is one of the most common deficiencies of micronutrients in children worldwide, which is highly associated with permanent cognitive-motor developmental delay, even after treatment [19]. These disorders can be attributed to different effects of iron deficiency on brain development [20]. One of the places that iron presents is in the hemoglobin structure, as it has a role in transporting oxygen to all tissues, like the brain. Iron-deficiency also causes metabolism reduction of some neurotransmitters, such as norepinephrine, dopamine, serotonin and gamma amino-butyric acid (GABA) [21]. Few studies have dealt with the relationship of other blood parameters with simple and complex FC. In this regard, some researchers have not found any relationship between anemia and FC [12], and some reported a link between iron-deficiency anemia and FC [22].

\section{Objectives}

The aim of this study is to compare anemia with Simple and Complex Febrile Seizures in children referred to the Ahvaz Golestan Hospital, Iran.

\section{Material and methods}

\section{Study design}

After obtaining permission from the Ethics Committee of Ahvaz Jundishapur University of Medical Sciences, Ahvaz, Iran (IR.AJUMS.REC.1395.491), and a written informed consent from the parents of patients, a cross-sectional, descriptive study was carried out on patients with febrile convulsion (FC) visiting the Pediatrics Ward of Ahvaz Golestan Hospital from 2013 to 2014.

\section{Setting, participants and variables}

In this study, 207 children aged 6 months to 5 years diagnosed with FC were selected as the sample. Patients diagnosed with meningitis, encephalitis, other CNS infections, electrolyte disorders and a history of epilepsy before FC were excluded from the study. To collect the required data and information from the medical records of patients, a checklist was developed based on the type of FC (simple or complex), which consisted of age, gender, frequency of seizures, family history, developmental status, hemoglobin $(\mathrm{Hb})$, hematocrit $(\mathrm{Hct})$, mean cell volume (MCV), mean cell hemoglobin (MCH), red blood cell distribution width (RDW), platelet count and red blood cell count (RBC).

\section{Statistical analyses}

In this study, we used the Kolmogorov-Smirnov test and found that distribution of data was normal. A descriptive statistics method, such as frequency tables, graphs and central indicators and dispersion, is used to describe the study variables, and the values are compared using the $t$-test. The descriptive statistics (mean and standard deviation) were used for data analysis and drawing the charts. In addition, the chi-square and independent $t$-test were used to compare the levels of significance and mean values between groups. All statistical analyses were performed using SPSS-17 at a significance level of 0.05 .

\section{Results}

\section{Descriptive data}

In this study, 207 children aged 6 months to 5 years were divided into two groups, namely simple and complex FC. According to the results, $151(73 \%)$ and $56(27 \%)$ patients suffered from simple and complex FC, respectively. In terms of gender, 128 patients $(61.8 \%)$ were male, and 79 of them (38.2\%) were female. This data indicates that there is no significant relationship between gender and type of FC $(p=0.27)$ (Table 1$)$.

\begin{tabular}{|c|c|c|c|}
\hline FC & Simple & Complex & Total \\
\hline Male & 90 (70.3\%) & 38 (29.7\%) & $128(100 \%)$ \\
\hline Female & 61 (77.2\%) & $18(22.8 \%)$ & 79 (100\%) \\
\hline
\end{tabular}

The mean age of patients with simple and complex FC was $26.93 \pm 20.073$ and $30.25 \pm 16.224$ months, respectively. There is no significant relationship between age and type of FC ( $p=$ 0.22 ). Moreover, the results showed that there is a significant relationship between the frequency of previous seizures and type of FC $(p<0.001)$ (Table 2$)$.

\begin{tabular}{|c|c|c|}
\hline & Simple & Complex \\
\hline 1 & 134 (88.7\%) & $27(48.2 \%)$ \\
\hline 2 & 12 (7.9\%) & $16(28.6 \%)$ \\
\hline 3 & $5(3.3 \%)$ & $9(16.1 \%)$ \\
\hline 4 & $0(0.0 \%)$ & $4(7.1 \%)$ \\
\hline Total & 151 (100\%) & 56 (100\%) \\
\hline
\end{tabular}

\section{Outcome data and main results}

According to the statistical analyses, family history affects the type of FC. In other words, patients with a history of simple $\mathrm{FC}$ in their families or relatives are more likely to suffer from FC than those with a history of complex FC in their families or relatives $(p=0.003)$ (Table 3$)$.

\begin{tabular}{|c|c|c|}
\hline & Simple & Complex \\
\hline No family history & $111(73.5 \%)$ & 38 (67.9\%) \\
\hline Father & 5 (3.3\%) & 5 (8.9\%) \\
\hline Mother & 4 (2.6\%) & 4 (7.1\%) \\
\hline Uncle & $4(2.6 \%)$ & 4 (7.1\%) \\
\hline Aunt & 1 (0.7\%) & 0 (0.0\%) \\
\hline Cousins & 19 (12.6\%) & $0(0.0 \%)$ \\
\hline Brother & 6 (4.0\%) & 5 (8.9\%) \\
\hline Sister & $1(0.7 \%)$ & $0(0.0 \%)$ \\
\hline Total & $151(100 \%)$ & 56 (100\%) \\
\hline
\end{tabular}

The mean values of $\mathrm{Hb}$ in patients with simple $\mathrm{FC}(10.85$ $\pm 0.84 \mathrm{~g} / \mathrm{dL})$ and complex FC $(10.41 \pm 1.13 \mathrm{~g} / \mathrm{dL})$ showed that there is no significant difference between these two groups of patients in this regard $(p=0.31)$. The same result was obtained for Hct, as the mean value of Hct for patients with simple and complex FC was $31.36 \pm 2.59 \mathrm{~g} / \mathrm{dL}$ and $32.28 \pm 2.28 \mathrm{~g} / \mathrm{dL}$, respectively $(p=0.85)$. 
The mean MCV in patients with simple FC and complex FC was found to be $74.47 \pm 6.05 \mathrm{fL}$ and $73.36 \pm 7.26 \mathrm{fL}$, respectively, which suggests there is no significant difference between the two groups of patients $(p=0.26)$. However, the results indicated that there is a significant difference between patients with simple FC and those with complex FC in terms of the mean $\mathrm{MCH}$ $(p=0.03)$, as $24.89 \pm 2.64 \mathrm{Pg}$ for simple FC and $23.98 \pm 2.85 \mathrm{Pg}$ for complex FC $(p=0.03)$ was obtained.

Based on the study results, the mean RDW in patients with simple FC $(15.03 \pm 2.08 \mathrm{~g} / \mathrm{dL})$ is higher than that of patients with complex FC (14.60 $\pm 1.08 \mathrm{~g} / \mathrm{dL})$; however, there is no significant difference between them $(p=0.051)$. Moreover, the blood platelet count showed a significant difference between patients with simple FC $(329.01 \pm 106.17 / \mu \mathrm{L})$ and those with complex FC $(273.38 \pm 85.99 / \mu \mathrm{L})(p=0.001)$.

The results also demonstrated that there is no significant difference between the two groups in terms of RBC, as its mean was $4.24 \pm 0.36 / \mu \mathrm{L}$ for simple $\mathrm{FC}$ and $4.35 \pm 0.47 / \mu \mathrm{L}$ for complex $\mathrm{FC}(p=0.08)$.

\section{Discussion}

FC is one of the most common seizures occurring during childhood [6]. This condition may be different in terms of age, gender and hemodynamic parameters, and it may cause simple and complex types. The present study aimed to investigate the status of CBC tests in FC patients with simple and complex types.

In the present study, the mean hemoglobin for all patients was $10.54 \mathrm{~g} / \mathrm{dL}$, which is indicative of anemia according to the laboratory scale described in the methodology. The results also show that the mean $\mathrm{Hb}$ was roughly the same in patients with simple and complex FC. This suggests an almost certain amount of hemoglobin in patients with anemic FC and no significant difference between the two groups in hemoglobin.

\section{Interpretation and generalizability}

A review of literature indicates that some other researchers have found similar results. Heydariyan and Vatankhah conducted a similar study on 240 patients to evaluate the role of anemia in two groups, patients with first episode of simple FC versus patients with febrile disease without $\mathrm{FC}$, and stated that there was no significant relationship between the mean $\mathrm{Hb}$ and simple $\mathrm{FC}$ [12]. Bidabadi and Mashouf conducted a study entitled "Relationship between Iron-Deficiency Anemia and First FC in the Case (Febrile Children without Convulsion) and Control (Healthy People of the Same Age and Gender) Groups" and reported that there was no significant relationship between the mean $\mathrm{Hb}$ in the two groups [15]. The same results were obtained by Sharif et al., who stated that there is no significant relationship between $\mathrm{Hb}$ and $\mathrm{FC}[21]$. It is noteworthy that the mean $\mathrm{Hb}$ of patients was very close in the present study and the three other studies mentioned above, and the study of variables of the same index in the above-mentioned studies indicates more confidence in consistent findings. What is evident in the field of research is that there are similar studies with opposite or contradictory results. Hence, some studies reporting inconsistent results with the findings of the present study can also be found in literature. For example, Özaydın et al. conducted a study on iron-deficiency anemia in children with simple and complex FC in Turkey and stated that there is a significant relationship between $\mathrm{Hb}$ and simple or complex FC [22]. This can be attributed to different disease conditions, depending on the geographic area. In the present study, patients with simple and complex FC (approx. 31\%) had similar HCT levels, which was significantly lower than the normal range in infants and children. The difference between patients with simple and complex FC was not statistically significant in terms of hematocrit. This is consistent with the findings of Bidabadi and Mashouf [15]. However, Yousefichaijan found a significant relationship between HCT and simple FC [23], which is inconsistent with the findings of the present study. Inconsistent results in different studies can be due to various reasons, such as different study populations and genetic susceptibility of individuals. $\mathrm{MCV}$ and $\mathrm{MCH}$ were lower than normal in all patients, with lower values in patients with complex FC compared to those with simple FC. Therefore, it can be concluded that the difference between the two groups was significant only in $\mathrm{MCH}$. Although patients with simple and complex FC were not different in hemoglobin, a significant difference was found between them in terms of the ratio of hemoglobin to red blood cells. Since there is no significant difference between patients with simple and complex FC in red blood cells, the importance of the difference between the two groups in the ratio of hemoglobin to red blood cells becomes more prominent. Derakhshanfar et al. [14] reported that there is a significant difference between patients with seizure and without seizure in $\mathrm{MCH}$. Özaydın et al. [22] found that there is a significant difference between patients with simple and complex FC in terms of $\mathrm{MCH}$. Although the findings of Heydarian and Vatankhah [12] and Bidabadi and Mashouf [15] showed that there is no significant difference between patients with simple and complex FC in $\mathrm{MCV}$ and $\mathrm{MCH}$, the results indicating the significant difference between them in terms of $\mathrm{MCH}$ and the ratio of hemoglobin to red blood cells can be a new and progressive finding in this regard. The results of the present study also showed that platelet count was lower in patients with simple FC, and there was no significant difference between the two groups. Özaydın et al. found that the difference between patients with simple and complex FC in terms of platelet count is statistically significant. Yousefichaijan et al. also reported a significant difference between patients with FC and the control group in terms of platelet count [23]. Therefore, it can be predicted that patients with complex FC are more vulnerable to life-threatening bleeding. Given the significance of the effect of family history on FC both in the present study and the study conducted by Sharif et al. [21], patients with a history of FC in their family and relatives should be further followed-up in terms of the status of platelet count and unusual inherited disorders.

\section{Limitations of the study}

As we used patients' profiles and documents that were recorded in hospital, due to an incomplete profile, we lost several patient details, and also because this study was conducted in only one center, the sample size and accessibility to patients were limited.

\section{Conclusions}

Based on the study results, it is recommended that patients with a history of FC in their family and relatives should be further followed-up in terms of the status of platelet count and unusual inherited disorders. In addition, considering the significance of MCV in patients with simple and complex FC, it is recommended that risk factors for FC be reduced and eliminated by changing eating habits and treatment programs.

Acknowledgments. The authors would like to thank the Ahvaz Jundishapur University of Medical Sciences, Ahvaz, Iran, for their support in this study and the staff of Clinical Research Development Unit of Golestan Hospital, Ahvaz Jundishapur University of Medical Sciences, Ahvaz, Iran, for their unwavering support during this research. 
Source of funding: This work was funded by the authors' own resources.

Conflict of interest: The authors declare no conflict of interests.

\section{References}

1. Momen AA, Davoodzadeh H, Morvaridnejad M. Evaluation of EEGs of children referred with first non-febrile seizure in Ahvaz, south west of Iran. Neuropsychiatr Neuropsychol 2017; 12(2): 49-53.

2. Offringa M, Newton R. Prophylactic drug management for febrile seizures in children. Evidence-Based Child Health: A Cochrane Review Journal 2013; 8(4): 1376-1485.

3. Paul SP. Clinical update: febrile convulsion in childhood. Community Pract 2012; 85(7): 36-38.

4. Patel N, Ram D, Swiderska N, et al. Febrile seizures. BMJ 2015; 351: h4240, doi: https://doi.org/10.1136/bmj.h4240.

5. Kumar B, Kalita J, Misra U. Febrile seizure. J Indian Med Assoc 2010; 108(7): 441-442, 451-456.

6. Miri Aliabad G, Khajeh A, Fayyazi A, et al. Clinical, epidemiological and laboratory characteristics of patients with febrile convulsion. J Compr Ped 2013; 4(3): 134-137.

7. Pal DK, Kugler SL, Mandelbaum DE, et al. Phenotypic features of familial febrile seizures Case-control study. Neurology 2003; 60(3): 410-414.

8. Amiri M, Farzin L, Moassesi ME, et al. Serum trace element levels in febrile convulsion. Biol Trace Elem Res 2010; 135(1-3): 38-44.

9. Nelson KB, Ellenberg JH. Predictors of epilepsy in children who have experienced febrile seizures. N Engl J Med 1976; 295(19): 1029$-1033$.

10. Graves RC, Oehler K, Tingle LE. Febrile seizures: risks, evaluation, and prognosis. Am Fam Physician 2012; 85(2): $149-153$.

11. Febrile seizures: long-term management of children with fever-associated seizures. Pediatrics 1980; 66(6): 1009-1012.

12. Heydarian $\mathrm{F}$, Vatankhah $\mathrm{H}$. The role of anemia in first simple febrile seizure in children aged 6 months to 5 years old. Neurosciences (Riyadh) 2012; 17(3): 226-229.

13. Shinnar S, Glauser TA. Febrile seizures. J Child Neurol 2002; 17(1 Suppl.): S44-S52.

14. Derakhshanfar $\mathrm{H}$, Abaskhanian A, Alimohammadi $\mathrm{H}$, et al. Association between iron deficiency anemia and febrile seizure in children. Med Glas (Zenica) 2012; 9(2): 239-242.

15. Bidabadi E, Mashouf M. Association between iron deficiency anemia and first febrile convulsion: a case-control study. Seizure 2009; 18(5): 347-351.

16. Hartfield DS, Tan J, Yager JY, et al. The association between iron deficiency and febrile seizures in childhood. Clin Pediatr 2009; 48(4): 420-426.

17. Kumari PL, Nair M, Nair S, et al. Iron deficiency as a risk factor for simple febrile seizures - a case control study. Indian Pediatr 2012; 49(1): 17-19.

18. El Kishawi RR, Soo KL, Abed YA, et al. Anemia among children aged 2-5 years in the Gaza Strip-Palestinian: a cross sectional study. BMC Public Health 2015; 15(1): 319, doi: 10.1186/s12889-015-1652-2.

19. Johnston MV. Iron deficiency, febrile seizures and brain development. Indian Pediatr 2012; 49(1): 13-14.

20. Carlson ES, Tkac I, Magid R, et al. Iron is essential for neuron development and memory function in mouse hippocampus. J Nutr 2009; 139(4): 672-679.

21. Sharif MR, Kheirkhah D, Madani M, et al. The relationship between iron deficiency and febrile convulsion: a case-control study. Glob Health Sci 2016; 8(2): 185-189.

22. Özaydın E, Arhan E, Cetinkaya B, et al. Differences in iron deficiency anemia and mean platelet volume between children with simple and complex febrile seizures. Seizure 2012; 21(3): 211-214.

23. Yousefichaijan $\mathrm{P}$, Eghbali $\mathrm{A}$, Rafeie $\mathrm{M}$, et al. The relationship between iron deficiency anemia and simple febrile convulsion in children. J Pediatr Neurosci 2014; 9(2): 110-114.

Tables: 3

Figures: 0

References: 23

Received: 14.06.2017

Reviewed: 24.06.2017

Accepted: 07.12.2017

Address for correspondence:

Prof. Ali Akbar Momen

Department of Pediatrics

Golestan Hospital

Ahvaz Jundishapour University of Medical Sciences

Ahvaz, Iran

Tel.: +98 6133334624

E-mail: momen_ali@yahoo.com 